\title{
New ant species of Myrmicaria Saunders, 1842 (Hymenoptera Formicidae) from Senegal, a second species with subpetiolar process in this genus
}

\author{
Antonio Scupola \\ Natural History Museum, Lungadige Porta Vittoria 9, 37129 Verona, Italy; e-mail: scupolant@ outlook.it
}

\begin{abstract}
The description of Myrmicaria morettoi n. sp. (Hymenoptera Formicidae), new ant species from Senegal is given. This species presents an unusual ring-like subpetiolar process as $M$. salambo Wheeler, 1922, the only species in the genus Myrmicaria Saunders, 1842 sharing the armed petiole with the taxon newly described here. A first report of M. salambo from Ivory Coast is also given.
\end{abstract}

KEY WORDS

Ants; Formicidae; Myrmicinae; Myrmicaria; new species; Senegal.

Received 02.10.2019; accepted 18.12.2019; published online 30.12.2019

\section{INTRODUCTION}

In the recent revision of the subfamily Myrmicinae (Ward et al., 2015), important taxonomic changes have been introduced and the most remarkable one was the reduction in number of tribes from twenty-five to only six. In this new context, the tribe Solenopsidini Forel, 1893 sensu novo also includes Myrmicaria Saunders, 1842, a genus with about 70 ant species, diffused from Africa to South East Asia, all characterized by antennae with only seven segments (scape included).

Myrmicaria has always been a neglected genus. For example, regarding the Afrotropical area only, just one revision was published (Santschi, 1925), a study that needs updates now; moreover of the about 30 African species, none has been described recently.

In my personal collection (ASPC), I keep a series of ant specimens belonging to an undetermined Myrmicaria from Senegal. In many of these specimens, the petiolar peduncle shows ventrally two extraordinarily developed teeth, often joined apically to form a great ring (Fig. 3). The presence of a sub- petiolar process is exceptional in the genus, and so far known only in the African M. salambo Wheeler, 1922. This last species slightly differs from my specimens, in the subpetiolar teeth less developed and (apparently) never joined apically, moreover the body is ochre in colour and the sculpture appears more areolate-reticulate, and the costumes are arboreal instead of terrestrial.

For all these reasons, I considered my Senegal's specimens as belonging to a new species that I describe here.

\section{MATERIAL AND METHODS}

The materials studied are deposited in the following private and public collections: ASPC (Antonio Scupola, personal collection, Italy), $\mathrm{MCZ}$ (Museum of comparative Zoology, Harvard University, USA); MSNV (Natural History Museum, Verona, Italy), MSNG (Natural History Museum, Genoa, Italy), MSNM (Natural History Museum, Milan, Italy). The measurements (min and max) ex- 
pressed in $\mathrm{mm}$ are taken by an ocular reticule mounted on Leica MB3 stereomicroscope at max $60 \mathrm{X}$ magnification. For brevity, in the text the following acronyms are used:

CL (cephalic length - the length of head, excluding the mandibles, measured from the midpoint of the anterior clypeal margin to the mid-point of the posterior margin in full-face view);

CW (cephalic width - maximum head width, measured immediately behind the eyes, with the head in full-face view);

FLD (frontal lobes distance - the maximum distance between the frontal lobes with the head in full-face view);

GL (gaster length - approximatively total length of gaster measured in lateral view);

MH (mesosoma width - the maximum height of the mesosoma, measured in lateral view from the dorsum of mesonotum to inferior edge of the mesopleuron);

ML (mesosoma length - the diagonal length of the mesosoma, measured in lateral view, from the angle at which the pronotum meets the cervix to the posterior basal angle of the metapleuron);

$\mathrm{PH}$ (petiole height - the maximum height of the petiole, measured in lateral view from the dorsum of petiolar node to the sternite (subpetiolar process excluded);

PL (petiole length - the length of the petiole, measured in lateral view from the anterior to posterior margin of the petiolar node);

PPH (postpetiole height - the maximum height of the postpetiole, measured in lateral view from the dorsum of postpetiolar node to the sternite);

PPL (postpetiole length - the length of the postpetiole, measured in lateral view, from the anterior to posterior margin of the postpetiolar node);

PPSD (pronotal-propodeal spine distance - the distance from the apex of the pronotal antero-lateral tooth to apex of propodeal spine in lateral view);

PSD (propodeal spines distance - the distance between the apex of the propodeal spines, with the propodeum in dorsal view);

SL (scape length - the maximum straight-line length of the scape, without the basal condyle);

SPSD (spiraculo - propodeal spine distance -the distance from the propodeal spiracle to the apex of the propodeal spine);

TL (total length - the approximate total length of body formed by the sum of CL+ML+PL+PPL+GL).
Indices. CI (cephalic index - CW/CLx 100); CS (cephalic size $-\mathrm{CW}+\mathrm{CL} / 2$ ); SI (scapus index CW/SLx100).

\section{RESULTS}

\section{Systematics}

Ordo HYMENOPTERA Linnaeus, 1758

Familia FORMICIDAE Latreille, 1809

Subfamilia MYRMICINAE Lepeletier de Saint-

Fargeau, 1835

tribus SOLENOPSIDINI Forel, 1893

genus Myrmicaria Saunders, 1842

Myrmicaria morettoi n. sp. (Figs. 1, 3, 4, 8)

EXAMINED MATERIAL. Holotypus (worker) (MSNV) and 13 Paratypes (workers) (ASPC, MSNG, MSNM): Senegal, Tambacounda, PN du Niokolo Koba, Assirik, loc. Ancien Poste du Mont Assirik, $144 \mathrm{~m}, 12^{\circ} 53^{\prime} 19^{\prime \prime} \mathrm{N} 12^{\circ} 43^{\prime} 10^{\prime \prime} \mathrm{O}, 21-$ 25.VII.2007, leg. Ph. Moretto \& F. Génier.

DESCRIPTION OF HOLOTYPE (worker). Colour: in dorsal view head, mesosoma and nodes of petiole and postpetiole black in colour. In dorsal view, first gastral tergite bicoloured (base and central part brownish-ochre, posterior brownish-black), remaining gastral segments brownish-black. In lateral view, head, mesosoma, petiolar peduncle ochre and nodes of petiole and postpetiole brownish-ochre. Mandibles ochre with masticatory margin brownish-black. Antennae brownish-black with apex of the last funicular segment indistinctly paler. Femora brown, tibiae brownish-ochre, tarsi much paler. Prosoma: in full-face view head sub-quadrate with sides convergent from the compound eyes to peristoma; posterior margin of head flat or weakly convex; maximum width of head before the anterior margin of compound eyes; the latter strongly protruding and placed near the corners of the posterior margin of head. Surface of head smooth and shiny with spaced longitudinal and sinuous carinae. A contiguous sinuous line of anastomosis on the front, in the vertex longitudinal carinae joined them to form a longer areolate sculpture. A median longitudinal and regular well-raised carina is present from frontal triangle to posterior margin of head; before the frontal triangle, this carina shortly enlarged with its 
internal space micro rugged. On clypeus, central carina present but less evident. Frons with 5-6 longitudinal carinae on each side of the median line. Antennal fossae deep with 3-4 circular carinae that surround the antennal sockets. In lateral view superior part of malar area with 4-5 longitudinal regular carinae, without anastomosis, the carinae reaching the compound eye and after the eye, they turn around and then come back to peristome. Clypeus with the raised median carina plus two lateral carinae placed in front the frontal lobes, also in the space between the median and the two lateral carinae are present 2-3 short basal carinulae. Mandibles triangular with 5-6 teeth, surface well striated with sparse subdecumbent setae. Antenna of 7 segments (scape, pedicel and five funicular segments). Second funicular segment longer than the first and the third together. Antennal club three-segmented with last segment much elongated. Scape smooth and shiny, weakly striated at the apex only. Mesosoma: surface smooth and very shiny. In dorsal view pronotum and mesonotum with a well-raised median regular carina and 4-5 weakly sinuous longitudinal carinae placed to each sides of the median carina. Space between median and first lateral carina very large and without anastomosis (except in the narrow space near the pronotal anterior margin). All longitudinal pronotal carinae in proximity of the anterior margin of pronotum merged by transverse, short anastomosis. Pronotum with anterolateral inferior tooth very prominent. In lateral view, pronotum with carinae and surface smooth, mesopleurae with regular car- inulae but surface rugose, metapleurae with carinulae but surface smooth. In lateral view, posterior part of mesonotum vertical and dorsally lobed and edged. In posterior view, vertical slope of mesonotum without sculpture except the median carina and the margins. In dorsal view, propodeum narrow and concave, lacking median carina and never with punctures or anastomosis, surface of the concavity very smooth and shiny. Propodeal spines elongated. Dorsum of propodeal spines sulcate. Slope of propodeum shorter that dorsal side. In lateral view propodeum with carinae. Propodeal spiracle circular and very prominent. Femora smooth and shiny, weakly striated at the apex only (in lateral view), raised granules at base of setae are also present. Metasoma: petiolar peduncle long, with ventral part armed with a subpetiolar process formed by two long translucent teeth curved at apex and joint apically together to form a ring. A linear translucent lamella is present along the ventral part of petiole. Postpetiole lacking anterior ventral process, therefore anterior ventral margin obtuse never prominent. In dorsal view dorsum of nodes of the petiole and postpetiole narrow laterally. In lateral view anterior and posterior sides of petiolar node weakly convergent to dorsum. In dorsal view, petiolar node with 4-6 spaced longitudinal carinae, postpetiolar node with 4-6 longitudinal carinae very close and joined with circular and regular pattern in the posterior slope. Base of gaster micro rugged and sub-opaque, remaining surface of gaster smooth and shiny. Pilosity: erect and sub-

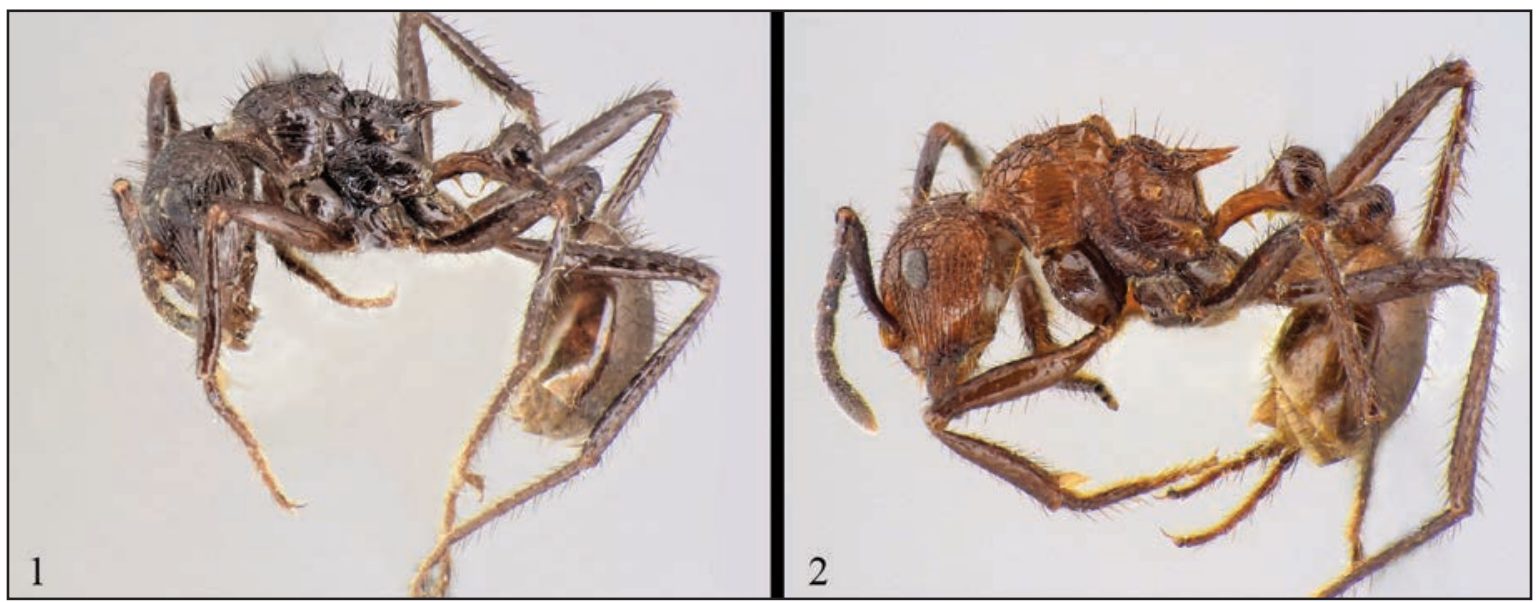

Figure 1. Myrmicaria morettoi n. sp., holotypus.

Figure 2. Myrmicaria salambo, habitus of specimen from Mbanto (Boundiali, Ivory Coast). 
erect setae only. Pubescence absent. All setae very long, stout and black in colour. Scape dorsally with long erect setae and shorter sub-erect setae, ventrally with short sub-erect setae only. In general, on the body setae always placed on carinae, while in the appendices each setae placed on a granule.

Holotypus measurements: CL: 1.558; CW: 1.517; SL: 1.599; FLD: 0.779; ML: 1.763; MH: 1.353; PPSD: 1.886; SPSD: 0.82; PSD: 0.451; PL: 0.943; PH: 0.533; PPL: 0.451; PPH: 0.574 ; GL: 2.01; CS: 1.537; CI: 97.368; SI: 94.87; TL: 6.725 .

VARIABILITY. Paratypes: subpetiolar process may vary in the development of the teeth as follow: teeth joined apically (ring-like) as the holotype in 5 workers; teeth aren't joint apically in 3 workers; teeth absent but a translucent narrow linear lamella along the venter of petiole it's well visible in 5 workers. Measurements: see Table 1.

ETYMology. The new species is dedicated to the French entomologist Philippe Moretto (valued specialist of scarabaeids Ontophagini), collector of the type specimens.

REMARKS. Myrmicaria morettoi $\mathrm{n}$. sp. is characterized by two longer teeth placed in the ventral part

\begin{tabular}{|l|l|l|}
\hline & $\begin{array}{c}\text { M. morettoi } \mathrm{n} . \mathrm{sp} . \\
\text { (n. 12) mm (min-max) }\end{array}$ & $\begin{array}{c}\text { M. salambo } \\
\text { (n. 7) mm (min -max) }\end{array}$ \\
\hline CI & $97.368-105.55$ & $96.770-117.50$ \\
\hline CL & $1.476-1.60$ & $1.517-1.984$ \\
\hline CS & $1.5170-1.608$ & $1.619-1.952$ \\
\hline CW & $1.504-1.616$ & $1.712-1.927$ \\
\hline FLD & $0.656-0.779$ & $0.738-0.82$ \\
\hline GL & $1.927-2.01$ & $2.132-2.46$ \\
\hline MH & $0.984-1.376$ & $1.025-1.189$ \\
\hline ML & $1.517-1.804$ & $1.763-1.968$ \\
\hline PH & $0.533-0.592$ & $0.533-0.615$ \\
\hline PL & $0.574-1.025$ & $1.066-1.517$ \\
\hline PSD & $0.416-0.56$ & $0.533-0.615$ \\
\hline PPH & $0.574-0.624$ & $0.656-0.779$ \\
\hline PPL & $0.451-0.624$ & $0.451-0.533$ \\
\hline PPSD & $1.744-2.091$ & $2.050-2.132$ \\
\hline SI & $94.87-100$ & $93.47-102.38$ \\
\hline SL & $1.558-1.696$ & $1.728-1.927$ \\
\hline SPSD & $0.656-0.820$ & $0.779-0.943$ \\
\hline TL & $6.00-7.80$ & $6.20-8.54$ \\
\hline & &
\end{tabular}

Table 1. Measurements of Myrmicaria morettoi n. sp. and $M$. salambo (see text) of petiolar peduncle, often bent and joined apically (Figs. 1, 3) to form a great ring-like process. A linear translucent linear lamella is also present. Until now, in the genus Myrmicaria, only M. salambo Wheeler, 1922, from Congo has a subpetiolar process (Fig. 5). Both species are distinguishable by the following characters:

Myrmicaria morettoi $\mathrm{n}$. sp.: size TL 6-7.8 mm. Brownish black in colour with petiolar peduncle and gaster castaneous or brownish-ocre. Clypeus with median carina absent or faintly traced. Femora shiny and weakly striated only at apex. Petiole with subpetiolar process formed by two long teeth joined apically (ring-like) (Fig. 3) or separate apically or totally absent in some cases (Fig. 4). Venter of postpetiole anteriorly obtuse (never angled or pointed). Pronotum and mesonotum with median raised carina, remaining carinae linear without anastomosis (except those on the anterior margin of pronotum). Dorsum of propodeum smooth and lacking longitudinal carinae or anastomosis. Nodes of peduncle carinated. Postpetiolar node with 4-6 longitudinal carinae, very close and in the posterior slope joined with circular and regular pattern.

Ecology: M. morettoi n. sp. nesting in the ground.

Myrmicaria salambo (Figs. 2, 5, 7, 9). TL 6.2$8.54 \mathrm{~mm}$. Orange-ochre or reddish-ochre in colour. Head and pronotum areolate-reticulated. Clypeus with shortly median carina. Femora subopaque and extensively striated. Petiole with subpetiolar process formed by two teeth always separate apically. Venter of postpetiole with a protrusion anteriorly obtuse (never angled or pointed). Pronotum and mesonotum with median raised carina, remaining carinae linear with evident anastomosis. Dorsum of propodeum with irregular carinae and anastomosis. Nodes of peduncle carinated.

Ecology: M. salambo is arboreal and attending climbing insects (Wheeler, 1922).

Concerning the measurements, M. morettoi $\mathrm{n}$. sp. has CW, SL, CI and CS relatively much smaller than $M$. salambo (see Table 1). The measures of $M$. salambo are based on 3 syntypes (MCZ) and 4 specimens from Ivory Coast, Boundiali, M'banto $09^{\circ} 35^{\prime} 18.1^{\prime \prime} \mathrm{N} 006^{\circ} 42^{\prime} 52.1^{\prime \prime} \mathrm{O}$, piège achatine, Moretto P. leg. (ASPC). This last datum represents the first citation from Ivory Coast and largely expands to the West the geographical distribution of M. salambo. 

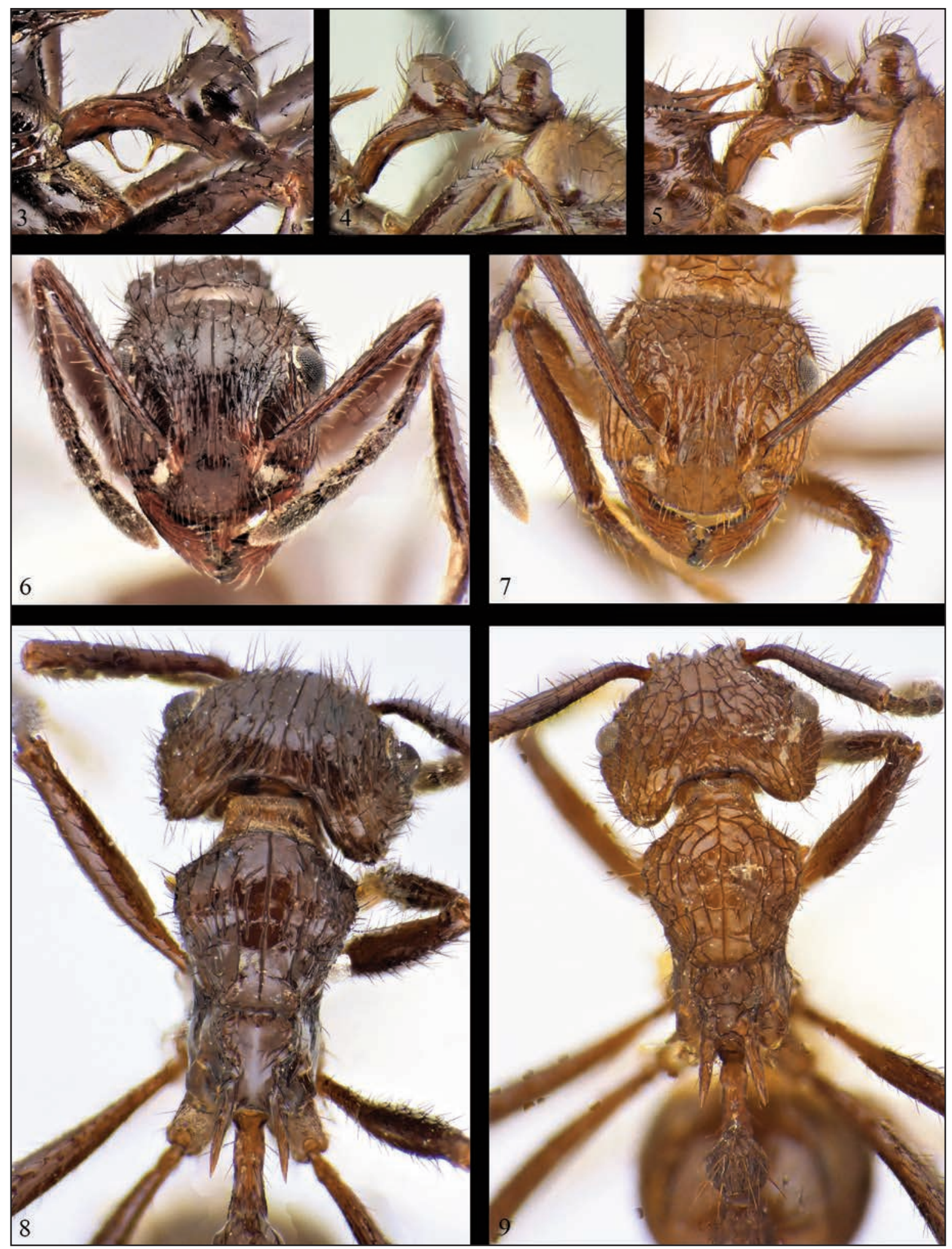

Figures 3-5: Ring-like supetiolar process in M. morettoi n. sp. (Fig. 3); unarmed petiole in M. morettoi n. sp. (Fig. 4); subpetiolar teeth in M. salambo (Fig. 5). Figures 6, 7. Head of M. morettoi n. sp. (Fig. 6); Head of M. salambo (Fig. 7). Figures 8, 9: Mesosoma of M. morettoi n. sp. (Fig. 8); Mesosoma of M. salambo (Fig. 9). 
I am not sure that the paratypes of $M$. morettoin. sp. lacking subpetiolar teeth (Fig. 4), come from the same nest as the holotype. However, in respect to the paratypes with armed petiole I did not find any differences in the body sculpture, colour, clypeus etc., and for these reasons I think they are probably all members of the same colony and consequently of the same new taxon.

It is obvious that the presence of the subpetiolar process in $M$. morettoi $\mathrm{n}$. sp. and in M. salambo easily distinguishes them from all other Myrmicaria (including Asian species). Some doubts raise about the aforementioned specimens of M. morettoi $\mathrm{n}$. sp. lacking subpetiolar teeth.

Therefore, I have examined the types of the other Central and Western African species, preserved in the Genoa Museum or photographed in the Antweb.org website. Below my results, where I emphasize the peculiar characters of each species, not present in the new species:

Myrmicaria exigua Andrè, 1890 sensu stricto and subspecies: reduced size TL 3-4.5 mm. Antenna not clubbed with only the last funicular segment elongated (in the other African Myrmicaria the antenna is clubbed in three segments).

Myrmicaria opaciventris Emery, 1893 sensu stricto and subspecies: body orange-ochre in colour. Surface of head and mesosoma opaque, gaster with half part of the first tergite opaque, very rugose or rugged, remaining tergites smooth. Femora well striated. Venter of postpetiole with a protusion anteriorly angled or pointed. Note that in the venter of petiole in the ssp. congolensis and ssp. mesonotalis it is present a translucent linear lamella well developed (as in M. morettoi n. sp. and M. salambo).

Myrmicaria baumi Forel, 1901 sensu stricto: dark reddish-brown in colour. Lateral and posterior part of head strongly reticulate lacking longitudinal carinae (present only between the frontal carinae). Dorsum of pronotum with transverse carinae. Mesonotum with longitudinal carinae but lacking median raised carina. Dorsum of propodeum with longitudinal carinae.

Myrmicaria baumi occidentalis Santschi, 1920: body castaneous in colour. Head as long as wide with longitudinal carinae but weakly reticulate. Clypeus without median carina. Pronotum and mesonotum with longitudinal carinae, curved near the anterior margin, without anastomosis except in the anterior pronotal margin). In the venter of peti- ole, a translucent longitudinal lamella is visible. Postpetiolar node with scarce longitudinal carinae and evidently spaced in the posterior slope (in $M$. morettoi $\mathrm{n}$. sp. the carinae in the posterior slope are very close together). Femora well striated. This subspecies is not related with M. baumi sensu stricto, but probably actually is a good species near to $M$. morettoi n. sp., (but different in head subsquare, femora and postpetiolar sculpture). Gaster evidently opaque as in $M$. opaciventris.

Myrmicaria distincta Santschi, 1925: head and mesosoma ochre in colour contrasting with the darkish-brown gaster. Pronotum with longitudinal carinae lacking anastomosis. Propodeum with some longitudinal carinae in dorsal view. Nodes of petiole and postpetiole smooth.

Myrmicaria distincta abissinica Santschi, 1925: body brownish-black in colour that contrasts with inferior part of head and pro-mesothorax reddishochre. Carinae on pronotum arched. Propodeum with short longitudinal carinae in dorsal view.

Myrmicaria distincta vorax Santschi, 1933: shape as $M$. distincta abissinica but much darker in colour. Carinae of pronotum arched. Propodeum dorsum with carinae and anastomosis in dorsal view.

Myrmicaria fumata Santschi, 1916 sensu stricto: body brown in colour. Dorsum of propodeum with longitudinal carinae and anastomosis in dorsal view. Femora well striated.

Myrmicaria fumata linearis Santschi, 1925: body darkish-ochre in colour. Head with numerous linear longitudinal carinae and some short anastomosis. Postpetiole with ventral protrusion angled anteriorly with the angle almost right. Dorsum of propodeum with longitudinal carinae in dorsal view.

Myrmicaria fusca Stitz, 1911: entire body surface smooth and polished. Face of head lacking longitudinal carinae except some short and fine carinae in the vertex, median carina visible between the frontal lobes only. In lateral view, sides of mesosoma and abdominal peduncle lacking longitudinal carinae. Dorsum of mesosoma lacking longitudinal carinae and anastomosis except for the median carina and two short oblique carinae placed at sides of the pronotum in dorsal view.

Myrmicaria striata Stitz, 1911: disc of pronotum with transverse carinae. Mesonotum with longitudinal carinae. Dorsum of propodeum with 1-2 short longitudinal carinae. 
Myrmicaria striata buttgenbachi Forel, 1913: body surface smooth and polished; general sculpture with longitudinal carinae regular linear but without anastomosis (except some very short anastomosis in the temples of head). Head with only 2-3 fine longitudinal carinae at sides of median carina. Pronotum with arched carinae, median carina not complete.

Myrmicaria striata insularis Santschi, 1920: head with longitudinal carinae irregular, interrupted, spaced and without anastomosis. Pedicel nodes smooth and shiny. Erect setae fine and yellow.

Myrmicaria irregularis Santschi, 1920: body surface smooth and polished and castaneous in colour. Head with 1-2 short carinae at side of the median carina in the frons area. Mesosoma with median carina and 2 or 3 longitudinal carinae lacking anastomosis. Venter of postpetiole with anterior process angled (similar in this character to $M$. opaciventris and M. fumata linearis).

Myrmicaria n. sp. of Senegal mentioned in Ants of Africa.org: body black in colour and much setose, with erect setae short and paler. Second and third funicular segments of the same length (in M. morettoi n. sp. they are different in length). Clypeus smooth showing only very faint traces of carinae (as $M$. morettoi n. sp.). Venter of petiole lacking teeth.

In conclusion, I think that M. baumi occidentalis is the taxon that more than others can be confused with the specimens of $M$. morettoi n. sp. lacking subpetiolar teeth, but the much spaced carinae present in the posterior side of postpetiole let immediately distinguish it.

\section{ACKNOWLEDGMENTS}

I am gratefully to following peoples: L. Toledano (MSNV) for a linguistic revision of the text; R. Poggi (MSNG) and S. Cover (MCZ Harvard University) for the loans of type material, P. Moretto (Toulon, France) collector of African ants in the field, and at last the anonymous referees.

\section{REFERENCES}

Santschi F., 1925. Révision des Myrmicaria d'Afrique. Annales de la Société entomologique de Belgique 64: 133-176.

Ward P.S., Brady S.G., Fisher B.L. \& Schultz T.R., 2015. The evolution of myrmicine ants: phylogeny and biogeography of a hyperdiverse ant clade (Hymenoptera: Formicidae). Systematic Entomology, 40: 61-81.

Wheeler W.M., 1922. The ants collected by the American Museum Congo Expedition. Bulletin of the American Museum of Natural History 45: 147-148, plate IX, figs. 1-2, text fig. 37 . 
